\title{
Single cell transcriptomic analysis of prostate cancer cells
}

\author{
Christopher J Welty ${ }^{1}, \|_{s a}$ Coleman², Roger Coleman², Bryce Lakely', Jing Xia², Shu Chen², Roman Gulati ${ }^{2}$, \\ Sandy R Larson ${ }^{1}$, Paul H Lange ${ }^{1,3}$, Bruce Montgomery ${ }^{4}$, Peter S Nelson ${ }^{2,4}$, Robert L Vessella ${ }^{3,1}$ \\ and Colm Morrissey ${ }^{1,5^{*}}$
}

\begin{abstract}
Background: The ability to interrogate circulating tumor cells (CTC) and disseminated tumor cells (DTC) is restricted by the small number detected and isolated (typically $<10$ ). To determine if a commercially available technology could provide a transcriptomic profile of a single prostate cancer (PCa) cell, we clonally selected and cultured a single passage of cell cycle synchronized C4-2B PCa cells. Ten sets of single, 5-, or 10-cells were isolated using a micromanipulator under direct visualization with an inverted microscope. Additionally, two groups of 10 individual DTC, each isolated from bone marrow of 2 patients with metastatic PCa were obtained. RNA was amplified using the WT-Ovation ${ }^{\mathrm{TM}}$ One-Direct Amplification System. The amplified material was hybridized on a 44K Whole Human Gene Expression Microarray. A high stringency threshold, a mean Alexa Fluor ${ }^{\circledR} 3$ signal intensity above 300, was used for gene detection. Relative expression levels were validated for select genes using real-time PCR (RT-qPCR).

Results: Using this approach, 22,410, 20,423, and 17,009 probes were positive on the arrays from 10-cell pools, 5cell pools, and single-cells, respectively. The sensitivity and specificity of gene detection on the single-cell analyses were 0.739 and 0.972 respectively when compared to 10-cell pools, and 0.814 and 0.979 respectively when compared to 5 -cell pools, demonstrating a low false positive rate. Among 10,000 randomly selected pairs of genes, the Pearson correlation coefficient was 0.875 between the single-cell and 5 -cell pools and 0.783 between the single-cell and 10-cell pools. As expected, abundant transcripts in the 5- and 10-cell samples were detected by RT-qPCR in the single-cell isolates, while lower abundance messages were not. Using the same stringency, 16,039 probes were positive on the patient single-cell arrays. Cluster analysis showed that all 10 DTC grouped together within each patient.
\end{abstract}

Conclusions: A transcriptomic profile can be reliably obtained from a single cell using commercially available technology. As expected, fewer amplified genes are detected from a single-cell sample than from pooled-cell samples, however this method can be used to reliably obtain a transcriptomic profile from DTC isolated from the bone marrow of patients with PCa.

Keywords: Prostate cancer, Single-cell, Transcriptome, Disseminated tumor cells

\footnotetext{
*Correspondence: cmorriss@uw.edu

'Department of Urology, University of Washington, Seattle, WA, USA

${ }^{5}$ Genitourinary Cancer Research Laboratory, Department of Urology,

University of Washington, Box 356510, Seattle, WA 98195, USA

Full list of author information is available at the end of the article
} 


\section{Background}

The development of novel technologies for capturing, enriching, and preserving exfoliated abnormal cells from body fluids or effusions as well as methods for concentrating the tumor-derived sub-cellular material for use in biomarker studies is currently a focus in the cancer field. Our laboratory and others have previously noted the presence of circulating tumor cells (CTC) in the blood and disseminated tumor cells (DTC) in the bone marrow of prostate cancer $(\mathrm{PCa})$ patients at time of radical prostatectomy, in post-radical prostatectomy patients with no evidence of disease, and in patients with advanced disease [1-4]. While men with DTC in the bone marrow are at an increased risk of future disease recurrence, many will not recur [2]. In addition, $\mathrm{PCa}$ is somewhat unique in the long delay that is often seen between the treatment of localized disease and the development of metastases. This suggests that tumor cells can remain dormant in the bone marrow for years and are subsequently "activated" by unknown mechanisms in some patients, leading to the formation of metastases [5-7]. However, to date there are no known markers to separate recurrent from indolent CTC/DTC or to distinguish dormant from senescent cells.

Transcriptomic analysis of CTC/DTC possesses the potential to identify markers that distinguish recurrent from indolent disease and genes important for tumor dormancy. However, using standard techniques this approach is limited by the need to pool multiple DTC to obtain enough mRNA for amplification $[1,2,5]$. This approach may combine dormant and indolent DTC, making identification of dormancy markers difficult or impossible. The primary challenge of single-cell transcriptome analysis is amplifying the mRNA to a detectable level while maintaining the correct sequences and relative signal intensities of the expressed genes.

Several methods for single-cell transcriptome analysis have been described. However, these methods are limited in their potential application due to the requirement for high throughput, time constraints, monetary considerations, and reproducibility. Therefore, we aimed to develop and validate an efficient, reproducible method for obtaining a transcriptomic profile from a single cell using commercially available technologies.

All single-cell transcriptome profiling methods rely on the creation and amplification of a cDNA library. To be detected using current technology, available mRNA from a single cell must be amplified approximately $10^{7}$-fold. There are two amplification strategies used: linear amplification through in vitro transcription (IVT) and exponential amplification through a PCR-based method [8-11]. The IVT method is more stringent and reduces the number of non-specific byproducts formed during amplification, but it is time intensive as each round of IVT only amplifies the available cDNA approximately 1000-fold. Through exponential amplification, PCR-based methods are more time efficient but are challenging to use with low abundance mRNA due to the potential for amplification of primer-primer dimers and loss of relative signal intensity through multiple rounds of amplification.

In the method described here, a commercial technology is used for amplification of low-abundance mRNA and a commercially available human oligonucleotide microarray to profile the transcriptome from a single PCa cell. Using clonally selected, synchronized single C4-2B PCa cells, pools of 5 cells, and pools of 10 cells, we determined that this method is efficient and effective but is limited predominantly by the abundance of the mRNA species available for amplification from a single cell. Herein we describe the usefulness and limitations of this approach.

\section{Methods}

\section{Culture and isolation of individual PCa cells}

To obtain a synchronized $\mathrm{PCa}$ cell population for analysis, we clonally selected C4-2B cells and cultured a single passage of cells in RPMI 1640 medium (Life Sciences Technologies Inc.) with 10\% FBS. Cells were treated with $30 \mathrm{mg} / \mathrm{ml}$ of Aphidicolin (Sigma) $24 \mathrm{~h}$ prior to isolation. Cells were trypsinized and resuspended in RPMI 1640 with $10 \%$ FBS. Ten replicates of single, pools of 5 , and pools of 10 cells (a total of 30 samples) were isolated with glass micropipettes using a TransferMan ${ }^{\circledR}$ NK micromanipulator (Eppendorf), lysed in a $2 \mu \mathrm{l}$ drop of WT-Ovation $^{\mathrm{TM}}$ One-Direct Amplification System lysis buffer (NuGEN) on a siliconized glass slide, and then stored for a minimum of 2 weeks at $-80^{\circ} \mathrm{C}$ before amplification. The transfer of cells to lysis buffer was verified by direct visualization.

\section{Isolation of individual DTC from the bone marrow of PCa patients}

All materials were acquired and used conforming with IRB-approved protocols at the University of Washington. DTC were isolated from bone marrow samples of PCa patients with advanced disease. Ten $\mathrm{ml}$ of bone marrow was aspirated from the iliac crest into a $30 \mathrm{ml}$ syringe containing $10 \mathrm{ml}$ of $6 \%$ sodium citrate. In samples obtained from patients, bilateral aspirates were obtained and combined for a total of $20 \mathrm{ml}$ of bone marrow. Samples were obtained using local anesthesia and taken from the posterior iliac crest. Processing of samples commenced within 1-2 hours and was completed within 5 hours.

\section{Cell enrichment}

Cell enrichment and isolation was performed as previously described [12]. Briefly, bone marrow aspirates were 
placed over a $15 \mathrm{ml}$ volume of Ficoll-Isopaque $1.077 \mathrm{~g} /$ $\mathrm{ml}$ (Accurate Chemical, Westbury, NY). Centrifugation subsequently yielded a mononuclear cell layer containing DTC, if present. The MACS system for immunomagnetic selection (Miltenyi Biotec, Auburn, CA) was then employed. Anti-CD45 and anti-CD61 antibodies were used for negative selection, targeting leukocytes, megakaryocytes, and platelets. Positive selection was then performed with immunomagnetic beads coated with anti-human epithelial antigen (HEA) antibodies.

\section{Identification of DTC}

For identification and isolation of DTC, the enriched population was subjected to immunostaining with fluorescein isothiocyanate labeled anti-BerEP4 antibodies (Dako, Carpinteria, CA) which bind a different epitope on HEA than the anti-HEA antibody used for positive selection. A phycoerythrin conjugated anti-CD45 antibody was also added for identification of leukocytes. The cells were kept on ice and viewed under fluorescent light using an inverted microscope. Individual cells were isolated using a micromanipulator, placed in $2 \mu \mathrm{l}$ of lysis buffer from the WT-Ovation ${ }^{\mathrm{TM}}$ One-Direct Amplification System (NuGEN) and stored at $-80^{\circ} \mathrm{C}$.

\section{Amplification of total RNA from PCa cells}

Total RNA was amplified from each sample using the WTOvation $^{\text {TM }}$ One-Direct Amplification System (NuGEN) according to the manufacturer's directions. The use of an aluminum cooling block on ice facilitated the handling of the reaction tubes. The amplified cDNA product purity and yield was determined by measuring the product's absorbance at 260, 280, and $340 \mathrm{~nm}$. The size distribution of the amplified cDNA product was analyzed on an Agilent Bioanalyzer using the RNA 6000 Pico LabChip with the mRNA Pico program. The mean fragment size was determined by creating a fragment on the electropherogram beginning at the start of the fluorescent signal and ending at a point corresponding to $50 \%$ of the total area. The end size (nt) value for this point was used to represent the mean fragment size. Post-SPIA modification and post-amplification work was performed in a separate workspace.

\section{Real-Time PCR (RT-qPCR)}

Amplified cDNA from each sample was used for RTqPCR amplification of 10 genes (Additional file 1: Tables S1). We measured relative gene expression changes in triplicate reactions using $5 \mathrm{ng}$ cDNA, 0.2 $\mu \mathrm{mol} / \mathrm{L}$ of each primer, and Power SYBR Green PCR master mix (Applied Biosystems) in a reaction volume of $10 \mu \mathrm{L}$ on a $7900 \mathrm{HT}$ Real-Time PCR machine (Applied Biosystems). All reactions were run in triplicate and assessed for quality and specificity by analysis of dissociation curves. We normalized mean quantification cycle value (Cq) for each gene to a housekeeping gene, RPL13A. We identified poor quality samples using Dixon's $Q$ test, a non-parametric test appropriate for small samples, to identify outliers based on the means of triplicate gene expression changes of four housekeeping genes (ACTB, RPL13A, YWHAZ, and GAPDH) [13].

\section{Labeling and hybridization of amplified material on Agilent chip}

Amplified cDNA from each sample and reference pool were labeled using the BioPrime ${ }^{\circledR}$ Total Genomic Labeling System (Invitrogen ${ }^{\mathrm{TM}}$ ). The reference pool was prepared by combining equal quantities of total RNA isolated from LNCaP, DU145, PC3, and CWR22 prostate epithelial cell lines grown at log phase, amplifying through two rounds using the MessageAmp ${ }^{\mathrm{TM}}$ II aRNA Amplification Kit $\left(\right.$ Ambion $\left.^{\circledR}\right)$, and converting to first strand cDNA. Hybridization probes were prepared by combining $6 \mathrm{mg}$ of Alexa Fluor ${ }^{\circledR} 3$ labeled sample and $1 \mathrm{mg}$ Alexa Fluor ${ }^{\circledR}$ 5 labeled reference and denatured at $95^{\circ} \mathrm{C}$ and hybridized at $63^{\circ} \mathrm{C}$ on Agilent Human $4 \times 44 \mathrm{~K}$ microarrays and processed following the manufacturer's specifications. Arrays were scanned on an Agilent DNA Microarray Scanner.

\section{Gene expression analysis}

Graphical examination of raw array signal indicated a batch effect due to subtle day-to-day variability in sample processing. We corrected for this effect by separately estimating mean Alexa Fluor ${ }^{\circledR} 3$ and mean Alexa Fluor ${ }^{\circledR} 5$ signal within single-, 5-, and 10-cell arrays between experimental days and adjusting values on the second day by the estimated difference. We normalized day-effect-corrected signals within arrays using loess and normal-exponential background correction with offset 50 and quantile normalized between arrays separately for single-, 5-, and 10-cell arrays using the Limma package in $R$ [14]. We then calculated sensitivity and specificity for the single-cell arrays using both the 5- and 10-cell arrays as the gold standard. Sensitivity is the proportion of commonly expressed probes in both array types out of total number of expressed probes in the gold standard. Specificity is the proportion of commonly unexpressed probes in both array types out of total number of unexpressed probes in the gold standard. Confidence intervals are based on a Bayesian approach using a uniform prior distribution. The same analysis was done for arrays from 5-cell pools using arrays from 10-cell pools as the gold standard. Relative signal intensity of individual markers was assessed using the M-ratio, defined as $\log _{2}$ (Alexa Fluor ${ }^{\circledR} 3 /$ Alexa Fluor $^{\circledR}$ 5), for all probes detected on all three sets of arrays, with detection defined as across-array-averaged, day-effect -adjusted, normalized Alexa Fluor ${ }^{\circledR} 3$ signal greater than 
300. We calculated Pearson correlation of the M-ratios between the single- and 5-cell, single- and 10-cell, and 5and 10-cell arrays. To assess the ability of the single-cell arrays to accurately order gene expression levels, we also calculated Spearman correlation of the ranks of M-ratios. The ability of the single-cell arrays to accurately detect the relative expression of probes on the same array was assessed by examining Pearson correlation of ratios of Mratios from 10,000 randomly selected probes between each pair of arrays. Gene set enrichment analysis was applied for a select set of genes in the single-, 5-, and 10-cell arrays using the mean centered $\log _{2}$ signal intensities $[15,16]$.

\section{Results}

\section{Quality control}

Cultured C4-2B cells, a PCa line derived from the LNCaP cell line, were grown, isolated, and amplified as illustrated (Figure 1). Quality of the amplified genetic material was assessed in four ways: purity and yield by spectrophotometer absorbance, gene detection by RTqPCR, efficiency of labeling amplified material prior to microarray hybridization, and standard deviation of oligo hybridization to microarrays. The range of $\mathrm{Abs}_{260} /$ $\mathrm{Abs}_{280}$ ratios of all samples was found to be 1.9 to 2.0, indicating high purity. The average amplified cDNA product yield for single-cell samples was $12.1+$ +/ $1.9 \mu \mathrm{g}$, for 5-cell samples $12.6+/-1.8 \mu \mathrm{g}$, and for 10-cell samples $13.5+/-2.3 \mu \mathrm{g}$. Yields are not expected to be directly proportional to input amount because, in the absence of template, one would expect nonspecific cDNA yields below 4 to $5 \mu \mathrm{g}$ of product. Using a Dixon's $\mathrm{Q}$ test we determined that there were no outliers for the single-, 5-, and 10-cell sample yields. Additionally, mean fragment sizes of the amplified products assessed on a Bioanalyzer were not significantly different between 1-, 5- and 10-cell samples. RT-qPCR was performed for 10 genes for all amplified samples. Specific genes and primers used are listed in Additional file 1: Table S1. Four housekeeping genes (ACTB, RPL13A, YWHAZ, and GAPDH), were reliably detected in the single-, 5-, and 10-cell samples. Based on the RT-qPCR results, we determined that one of the single-cell samples and one of the 10-cell samples was a failure (Additional file 2: Table S2). In addition, we determined that the Cq for individual cells for housekeeping genes was higher than that of $10 \mathrm{pg}$ of RNA from the same clonal population suggesting less material was available for amplification from a live single cell isolated from culture (Additional file 2: Table S2). For microarray analysis, amplified cDNA from the single-, 5-, and 10-cell samples and from a reference pool were labeled using the BioPrime ${ }^{\circledR}$ Total Genomic Labeling System (Invitrogen ${ }^{\mathrm{TM}}$ ). Labeling efficiency was defined as the amount of Alexa Fluor ${ }^{\circledR} 3$ label used of amplified RNA. Labeling efficiency was $5.2 \pm 1.4 \mathrm{pmol} / \mathrm{ng}$ for the single-cell samples, $6.3 \pm 1.7$ $\mathrm{pmol} / \mathrm{ng}$ for the 5-cell samples, and $8.6 \pm 1.6 \mathrm{pmol} / \mathrm{ng}$ for the 10-cell samples. This indicates that RNA quality was similar for all three samples, but labeling efficiency was higher with larger numbers of cells. Once again, a Dixon's Q test was used to identify potential outliers based on labeling efficiency and no significant outliers were found. To determine if poor quality samples could be identified based on Alexa Fluor ${ }^{\circledR} 3$ intensity, we analyzed the changes of distributions of $\log 2$ (Alexa Fluor $^{\circledR} 3$ ) for the 10 replicates in the single-, 5-, and 10-cell samples. Using a Dixon's Q test based on 25th, 50th, and 75th percentiles, maximum values, and mean absolute deviations to quantify array performance, we did not identify additional poor quality samples. The samples deemed to be of poor quality by any of the four criteria (purity and yield by spectrophotometer absorbance, gene detection by RT-qPCR, efficiency of labeling amplified material prior to microarray hybridization, or the standard deviation of oligo hybridization to microarrays) were excluded from further analyses.

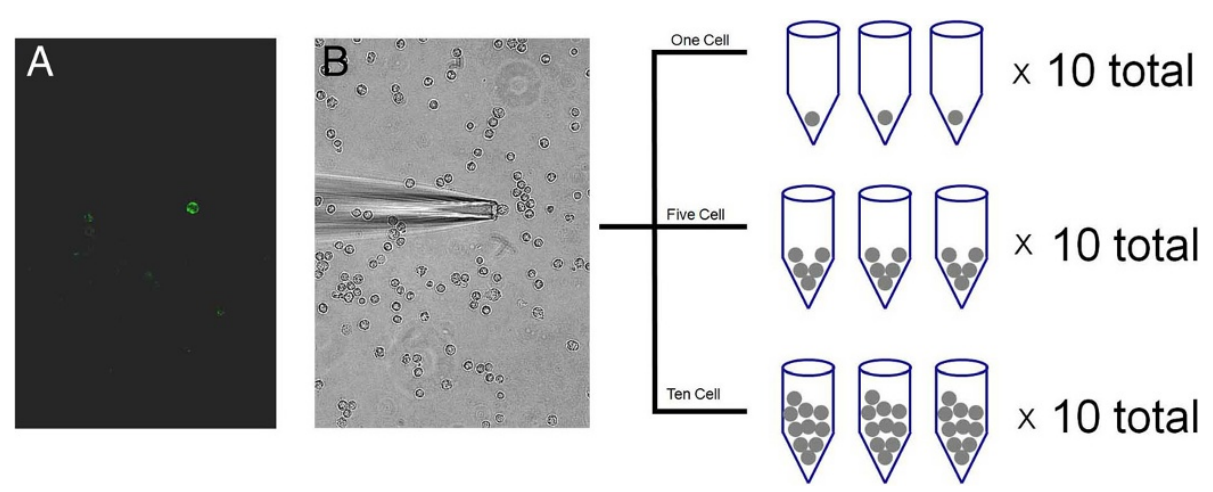

Figure 1 Isolation of individual cells. A. Fluorescently labeled EpCAM positive cells are identified and B. Under light microscopy a single C4-2B cell is harvested using a micropipette system (40X). 


\section{Microarray results}

Amplified and Alexa Fluor ${ }^{\circledR} 3$ labeled single-, 5-, and 10-cell samples were hybridized to Agilent $4 \times 44 \mathrm{k}$ human genome microarrays along with Alexa Fluor ${ }^{\circledR} 5$ labeled reference RNA (Figure 2). A total of 38,695 probes were present on each array. Previously, we obtained 24,885 positive probes on a reference array, which represents the average number of probes detected from C4-2B cells using standard mRNA isolation, amplification, and array protocols. In this study, 17,009 probes were positive on the single-cell arrays, 20,423 probes on the arrays from 5 -cell pools, and 22,410 probes on the arrays from 10 cell pools. The sensitivity and specificity of gene detection on the arrays from single-cell pools were 0.739 and 0.972 respectively when compared to arrays from 10-cell pools, and 0.814 and 0.979 respectively when compared to arrays from 5-cell pools, demonstrating a low false positive rate.

\section{Relative signal intensity}

The Pearson correlation between M-ratios on the arrays from single-cell and 5-cell pools was 0.876 and on the arrays from single-cell and 10-cell pools was 0.787 . The Spearman correlation between ranks of M-ratios on the arrays from single-cell and 5-cell pools was 0.842 and on the arrays from single-cell and 10-cell pools was 0.786 . To test whether gene-to-gene ratios were maintained across sample type we randomly selected 10,000 pairs of genes as an approximation to all 133 million pairs of genes, which we refer to as differences of M-ratios. The Pearson correlation of differences between M-ratios on the arrays from cells single and 5-cell pools was 0.856 and on the single-cell and 10-cell pools was 0.780 . Figure 3 shows the global correlation of $\mathrm{M}$-ratios and difference of M-ratios between single-cell and 5-cell and single-cell and 10-cell pools. We also conducted an Mratio pair-wise correlation analysis for the single cell to gauge the presence of outliers. Based on the correlation analysis, we could not find any outliers for the single

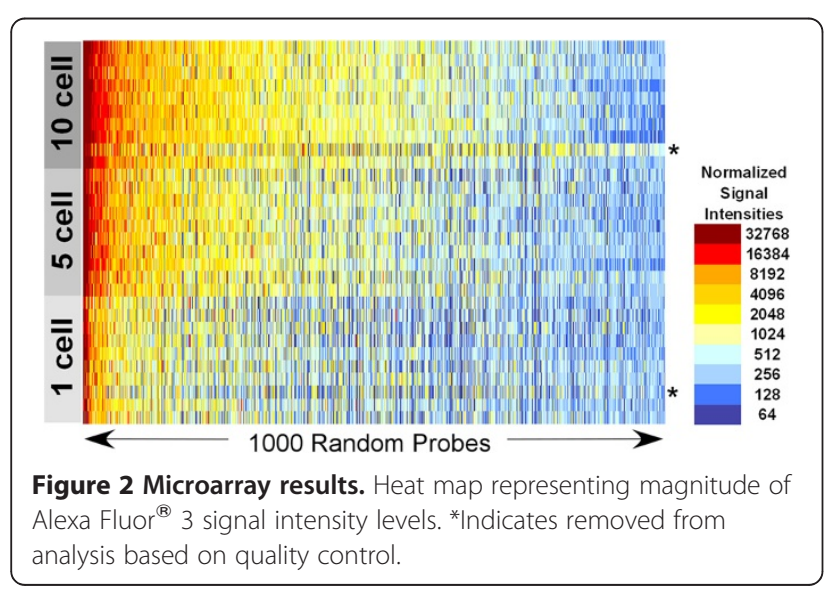

cells (Additional file 3: Table S3). The strong positive correlations between M-ratios, ranks of M-ratios, and ratios of M-ratios on the single-cell analyses relative to the 5- and 10-cell pools indicates that signal intensities, their ordering, and their relative levels are generally preserved in the arrays from single-cells. Furthermore the average 10-cell profile versus each individual cell profile the pair-wise correlation coefficient ranged from 0.537 to 0.787 , suggesting limited variability between the 1 cell profiles.

\section{RT-qPCR results}

To determine if RT-qPCR could validate the results of the gene expression arrays, we assessed the expression of one epithelial (EPCAM) and five well known prostate epithelial associated genes. We compared the expression levels relative to RPL13A in each of the single-, 5-, and 10-cell samples as well as 10 and $100 \mathrm{pg}$ of C4-2B RNA from the same culture that the other $\mathrm{C} 4-2 \mathrm{~B}$ cells were isolated from (Figure 4). For the androgen receptor (AR), epithelial cell adhesion molecule (EPCAM), and prostate-specific antigen (KLK3), we observed consistency between the single-, 5-, and 10-cell samples. However, there was a decrease in expression levels for TMPRSS2, FK506-binding protein 5 (FKBP5), and prostatic acid phosphatase (ACPP) respectively in the single-cell samples relative to the 5- and 10cell samples. Additionally FKBP5 was not detected in five 5-cell samples and ACPP was not detected in six 5-cell samples and four 10-cell samples (Figure 4).

\section{Comparison of microarray signal intensity to RT-qPCR}

We wished to determine an approximate cut off that would indicate when there is a loss of fidelity between the oligo gene expression array Alexa Fluor ${ }^{\circledR} 3$ signal intensity and RT-qPCR. Our results suggest that an Alexa Fluor ${ }^{\circledR} 3$ signal intensity above the $75^{\text {th }}$ percentile would be an approximate cut off that we could use to validate our results using RT-qPCR for the C4-2B samples, recognizing that there is inconsistency between the oligo labeling on the array and the amplicons obtained using RT-qPCR (Tables 1 and 2). Nonetheless this cutoff is a useful indicator of fidelity.

\section{Gene set enrichment analysis}

To determine which biological pathways were consistent between the single-, 5-, and 10-cell C4-2B samples, we performed gene set enrichment analysis. The top 25 biological pathways are listed in Additional file 4: Table S4 with a FDR q-value $<0.0001$. As expected, the top 25 pathways are associated with RNA processing, protein translation, and mitochondrial activity. The Pearson correlations for the normalized enrichment scores were significant at 0.932 and 0.923 for single- versus 5- and 

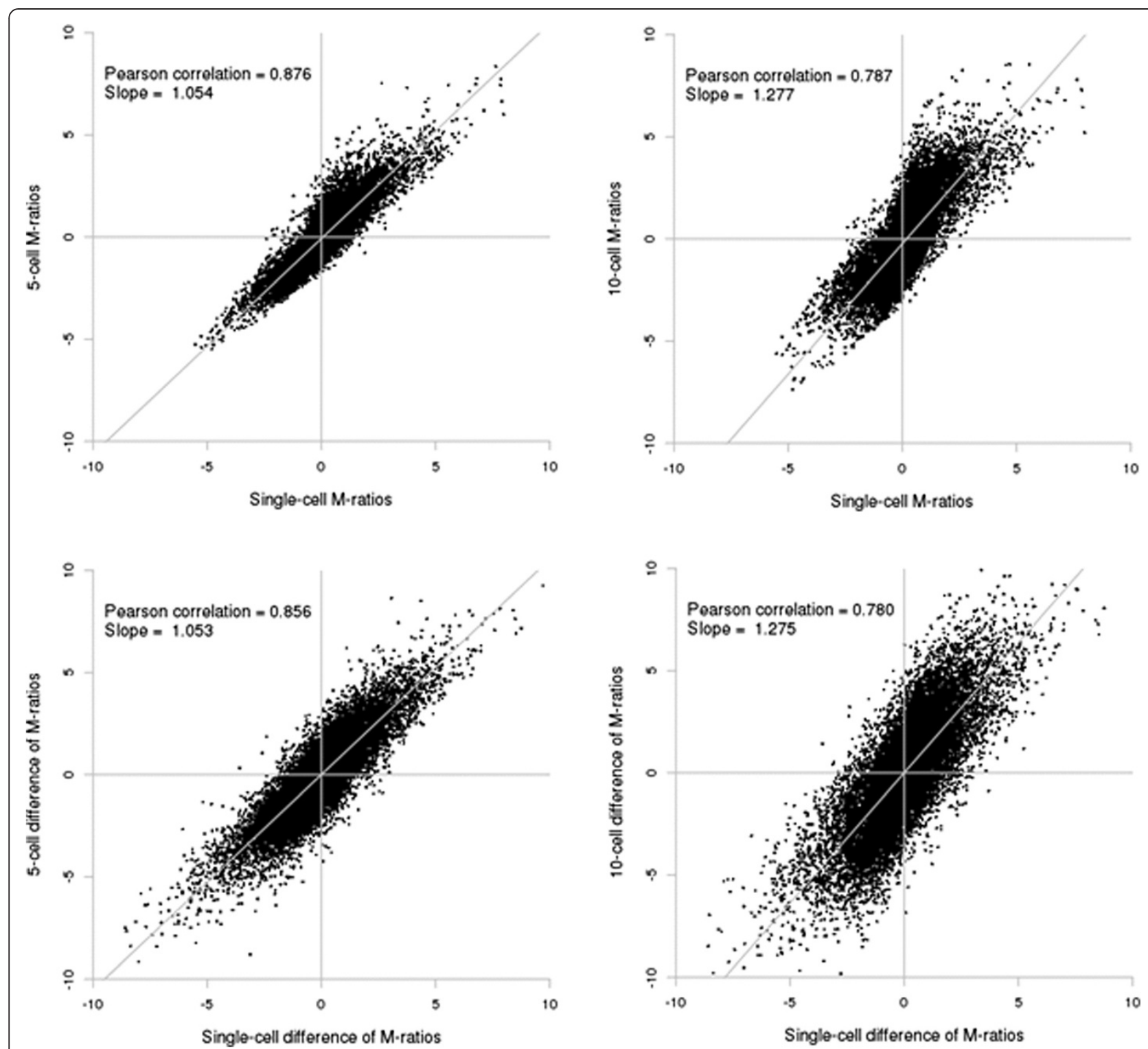

Figure 3 Correlation of M-ratios (top two panels) and difference of M-ratios (bottom two panels) between arrays. The mean M-ratio for each probe detected on the single-cell arrays was compared to the M-ratio for the same probe on the 5- or 10-cell array. The M-ratio is defined as $\log _{2}$ (Alexa Fluor ${ }^{\circledR}$ 3/Alexa Fluor ${ }^{\circledR}$ ).

10-cell samples respectively and 0.946 for 5 - versus 10 cell samples.

The transcriptomic analysis of patient DTC

To validate the method we wished to determine if the methodology could be used in the analysis of individual tumor cells isolated from the bone marrow of patients. Similar to the C4-2B cell samples, 10 individual DTC from 2 patients were amplified. To determine if there was a difference in the input material from the patients relative to the cultured cells we assessed the amplified fragment sizes. The mean fragment size on the
Bioanalyzer for 1 C4-2B cell was 610 (range 275-966), 5-cell 493 (range 144-968) and 10-cell 598 (range 1781168). For the patient cells it was 193 (range 139-319) and 224 (range 127-469) respectively (we did not observe any associations between amplicon size and the gene expression array results). After amplification the samples were Alexa Fluor ${ }^{\circledR} 3$ labeled single-cell samples were hybridized to Agilent $4 \times 44 \mathrm{k}$ human genome microarrays along with Alexa Fluor ${ }^{\circledR} 5$ labeled reference RNA. A total of 16,039 probes were positive on the patient sample arrays. Cluster analysis revealed similarities between the cells isolated within a patient, but not 
ACPP

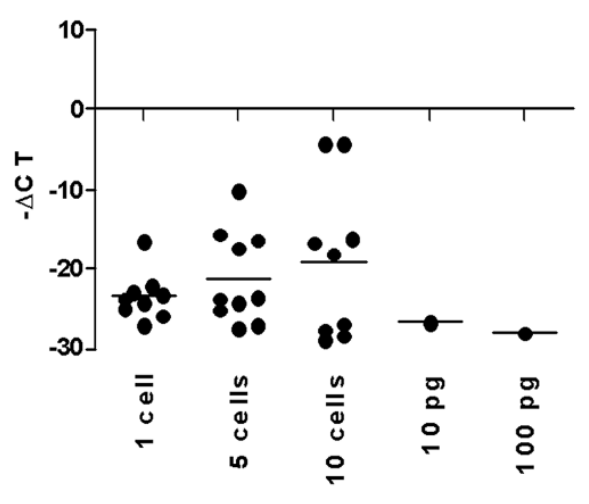

EPCAM

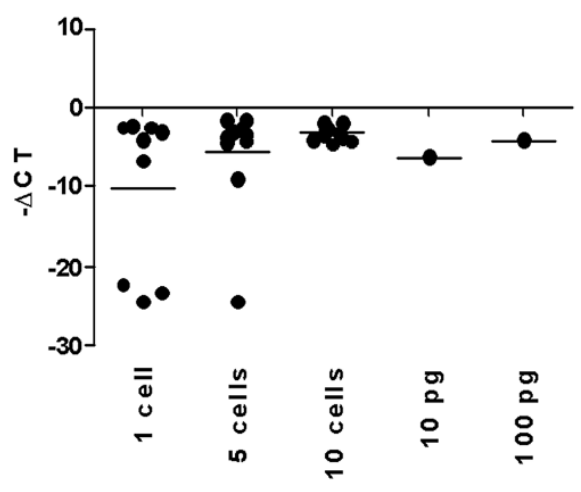

TMPRSS2

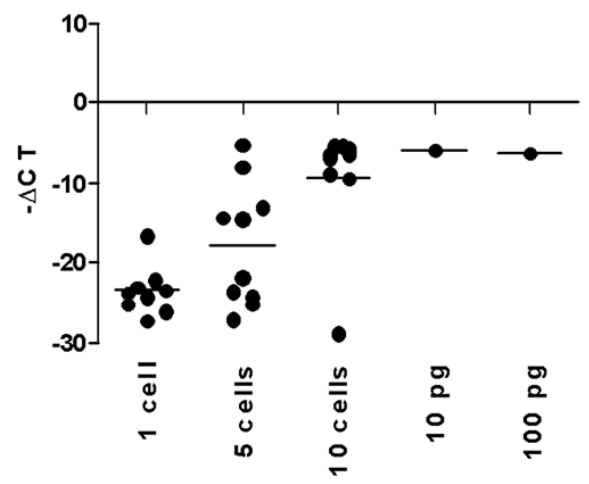

AR

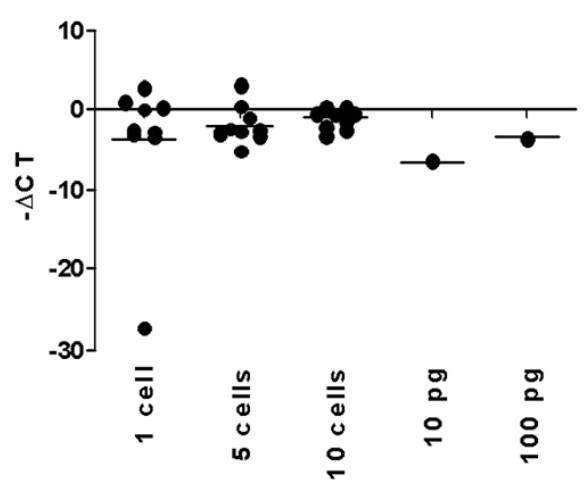

FKBP5

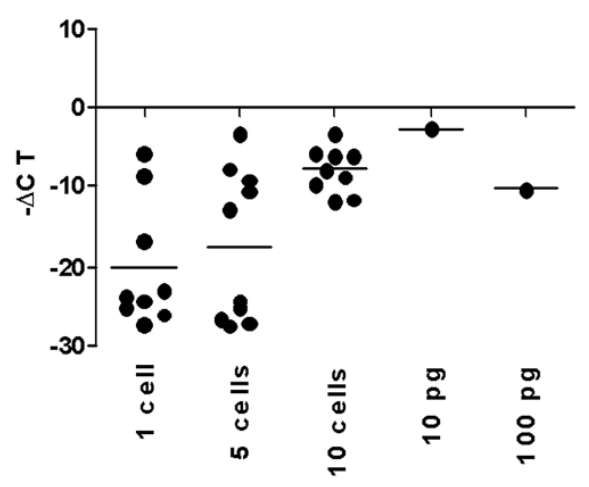

KLK3

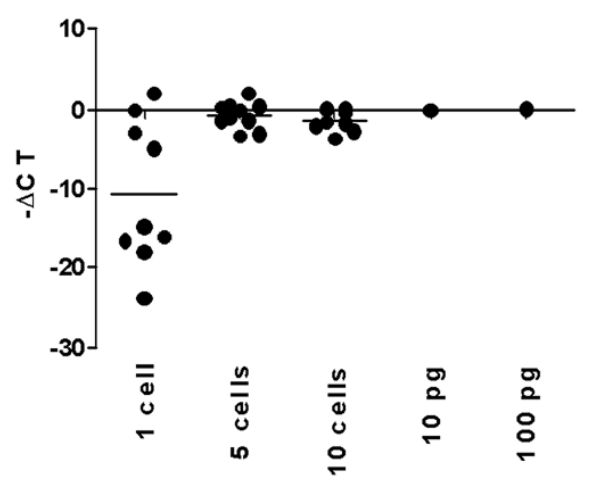

Figure 4 Real-time PCR results. RT-qPCR results for AR, EPCAM, KLK3, TMPRSS2, FKBP5 and ACPP. Inverse cycle thresholds relative to RPL13A are shown for single-cell, 5-cell, 10-cell samples as well as $10 \mathrm{pg}$ and $100 \mathrm{pg}$ and unamplified of C4-2B total RNA from the same original culture.

between patients (Figure 5). We then analyzed the expression of prostate specific genes from each of 10 individual DTC isolated from the two PCa patients (2613 and 2679). The heatmap in Figure 6 shows that each of the top 10 expressed prostate specific genes was in the top $75^{\text {th }}$ percentile of genes expressed in these samples. Additionally, to identify differences between biological pathways present in the two patient sample sets, using 10,731 known genes to compare the profiles of each patient by gene set enrichment analysis two gene sets were significantly upregulated with a false discovery rate $<25 \%[15,16]$. The two pathways were the TRKA pathway (binds nerve growth factor and is implicated in PCa proliferation) and the activation of the RAC pathway (associated with cell motility).

\section{Discussion}

Herein we describe a one-step amplification of mRNA from a single cancer cell and hybridization to an 
Table 1 Detection of six selected genes by RT-qPCR from a total of 9 samples in the 1 and 10 cell category and 10 samples in the 5 cell category

\begin{tabular}{|c|c|c|c|c|c|c|}
\hline \multirow[b]{2}{*}{ Gene } & \multicolumn{3}{|c|}{ \# of samples detected by RT-qPCR } & \multicolumn{3}{|c|}{ Rank percentile ( $n=38695$ probes) } \\
\hline & 1 -cell $(n=9)$ & 5 -cell $(n=10)$ & 10 -cell $(n=9)$ & 1 -cell $(n=9)$ & 5 -cell $(n=10)$ & 10-cell $(n=9)$ \\
\hline$A R$ & 8 & 10 & 9 & 99 & 99 & 99 \\
\hline KLK3 & 6 & 10 & 9 & 94 & 98 & 95 \\
\hline EPCAM & 6 & 9 & 9 & 92 & 95 & 95 \\
\hline TMPRSS2 & 0 & 4 & 8 & 85 & 89 & 86 \\
\hline FKBP5 & 2 & 5 & 9 & 69 & 60 & 63 \\
\hline ACPP & 0 & 4 & 5 & 50 & 45 & 54 \\
\hline
\end{tabular}

Detection was considered as having an inverse Cq of at least -20 relative to RPL13A.

oligonucleotide gene expression array using commercially available gene expression array technology. The amplification technology employed used a linear isothermal method to amplify mRNA. Unlike exponential amplification, this linear amplification approach is carried out by replication of only the original transcripts not replication of copies. An additional advantage is that the amplified cDNA can be used directly in RT-qPCR analysis. The commercially available kit used was employed based on availability at the start of these studies and does not compare this to other now commercially available kits using a similar platform. Using this microarray platform, approximately $76 \%$ of the probes detected in 10 -cell pooled samples were detected in the single-cell samples. This decrease in probes detected and signal intensity appears to be related to the amount of material available. For those probes that were detected, we found a high correlation between the relative signal intensity of detected probes on arrays from single-cell and 10-cell pools. Additionally, we found a significant correlation between the single-, 5-, and 10-cell samples using gene set enrichment analysis.

Many groups have used PCR-based technologies to analyze a subset of genes from a single cell; however, screening of thousands of genes is more challenging. Other groups, notably Kurimoto et al. [10], have isolated a transcriptomic profile of individual cells; these methods are extremely effective but are not always available throughout the greater scientific community and are labor and time intensive. Similar methods to profile individual cells are also being developed; Islam et al. [17] have used highly multiplex RNA-seq to obtain a singlecell transcriptomic profile on a large number of single cells. Reproducibility was good; however, as in our study, the detection level decreased for genes expressed at lower levels. Additionally, they found a correlation of 0.86 for embryonic stem cells and 0.63 for embryonic fibroblasts compared to 0.86 for $10 \mathrm{pg}$ of human brain RNA as a reference. Cann et al., have also used mRNASeq to profile gene expression in CTC [18]. Using Magsweeper technology to isolate $\mathrm{LNCaP}$ cells they found a high correlation in gene expression $\left(R^{2}=0.985\right)$ between Magsweeper and control pools of LNCaP cells. For LNCaP controls they measured $4622 \pm 136$ RefSeq transcripts with $\geq 10$ FPKM. Of 67 CTC from 13 patients, 20 CTC from 4 patients had $2362 \pm 865$ RefSeq transcripts with $\geq 10$ FPKM. Using an unsupervised cluster analysis the authors state that only two of 20 CTC did not cluster with other CTC from the same patient suggesting limited heterogeneity. However this may be limited by the number of samples tested and the number of transcripts available for analysis. While a smaller number of transcripts were available for analysis using this mRNA-Seq approach compared to the gene

Table 2 Detection of six selected genes by RT-qPCR from a total of 9 samples in the 1 and 10 cell category and 10 samples in the 5 cell category

\begin{tabular}{|c|c|c|c|c|c|c|}
\hline \multirow[b]{2}{*}{ Gene } & \multicolumn{3}{|c|}{ \# of samples detected by RT-qPCR } & \multicolumn{3}{|c|}{ Rank percentile ( $n=38695$ probes) } \\
\hline & 1 -cell $(n=9)$ & 5 -cell $(n=10)$ & 10-cell $(n=9)$ & 1 -cell $(n=9)$ & 5 -cell $(n=10)$ & 10-cell $(n=9)$ \\
\hline$\overline{\mathrm{ACTB}}$ & 7 & 7 & 9 & 100 & 100 & 100 \\
\hline GAPDH & 8 & 10 & 9 & 94 & 94 & 90 \\
\hline YWHAZ & 8 & 10 & 9 & 97 & 98 & 98 \\
\hline RPL13A & 9 & 10 & 9 & 99 & 100 & 100 \\
\hline
\end{tabular}

Detection was considered as having a Cq of $<30$. Rank percentile represents the average Alexa Fluor ${ }^{\circledR} 3$ intensity from all oligonucleotides on the array that passed quality control for a given gene with outliers excluded using Dixon's $Q$ test. $n=$ number of samples analyzed. 


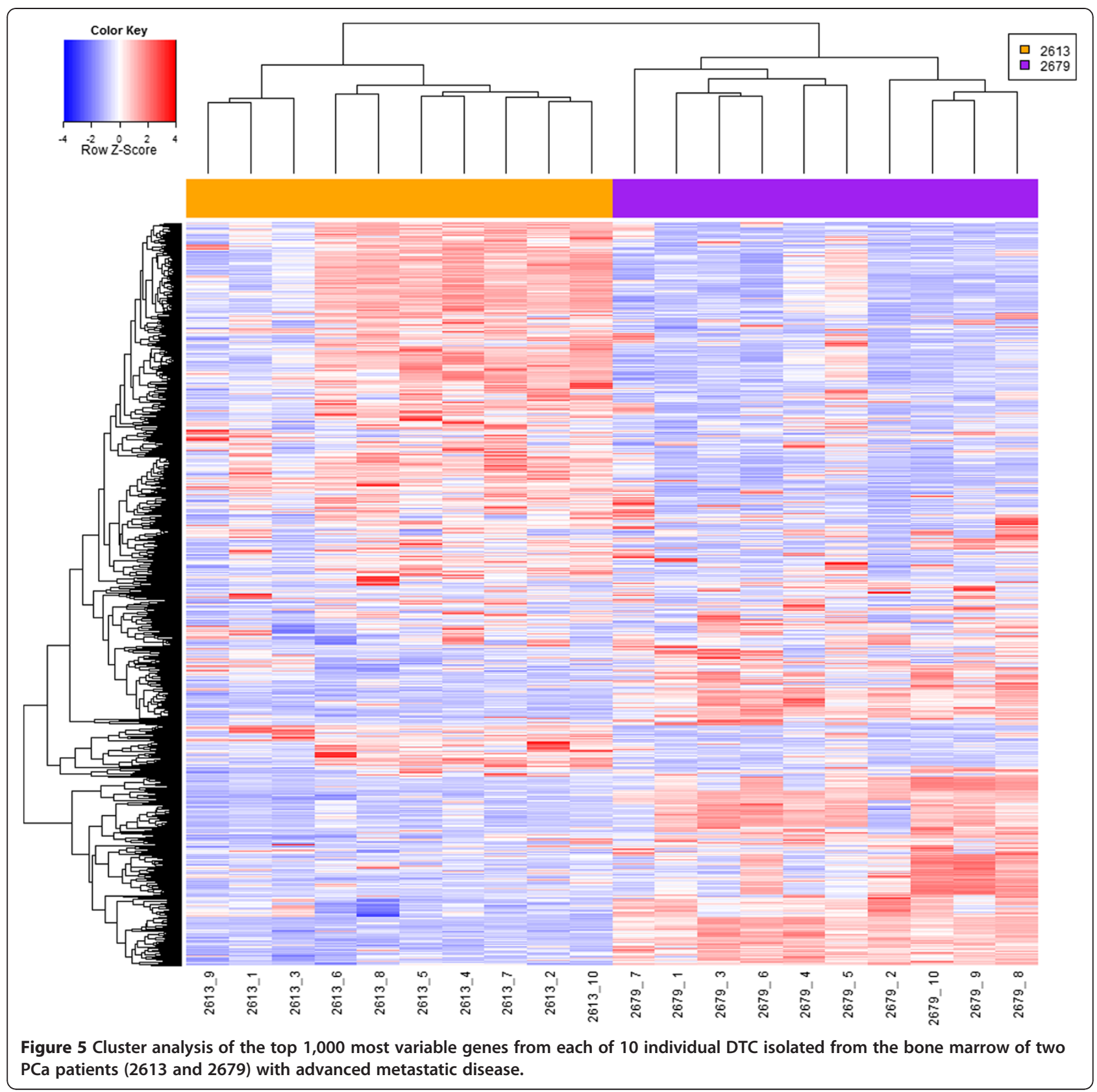

expression arrays reported here, there are other obvious benefits to using mRNA-Seq relative to gene expression analysis alone.

The importance of using a commercially available technology accessible to a broad user group is important as it provides the ability to examine individual cells to most laboratory based research groups. This is of particular interest to groups working on CTC/DTC, where the technology needs to go beyond measuring cell number to characterizing the molecular profile of individual cells $[7,19]$. This is particularly important as tumor cell heterogeneity could significantly impact the transcriptomic profile displayed by these cells at different times throughout the disease process [20]. Tumor cell heterogeneity may arise due to clonal evolution within a single tumor or multiple metastatic tumor sites shedding cells. This heterogeneity restricts our ability to obtain a definitive transcriptomic profile from CTC/DTC to further characterize the biological events occurring in response to treatment or the identification of tumor biomarkers. We have shown that a commercially available technology could be used to profile tumor cells grown in culture and cells isolated from the bone marrow of patients with PCa. These data show that the technology is available to obtain a significant and 


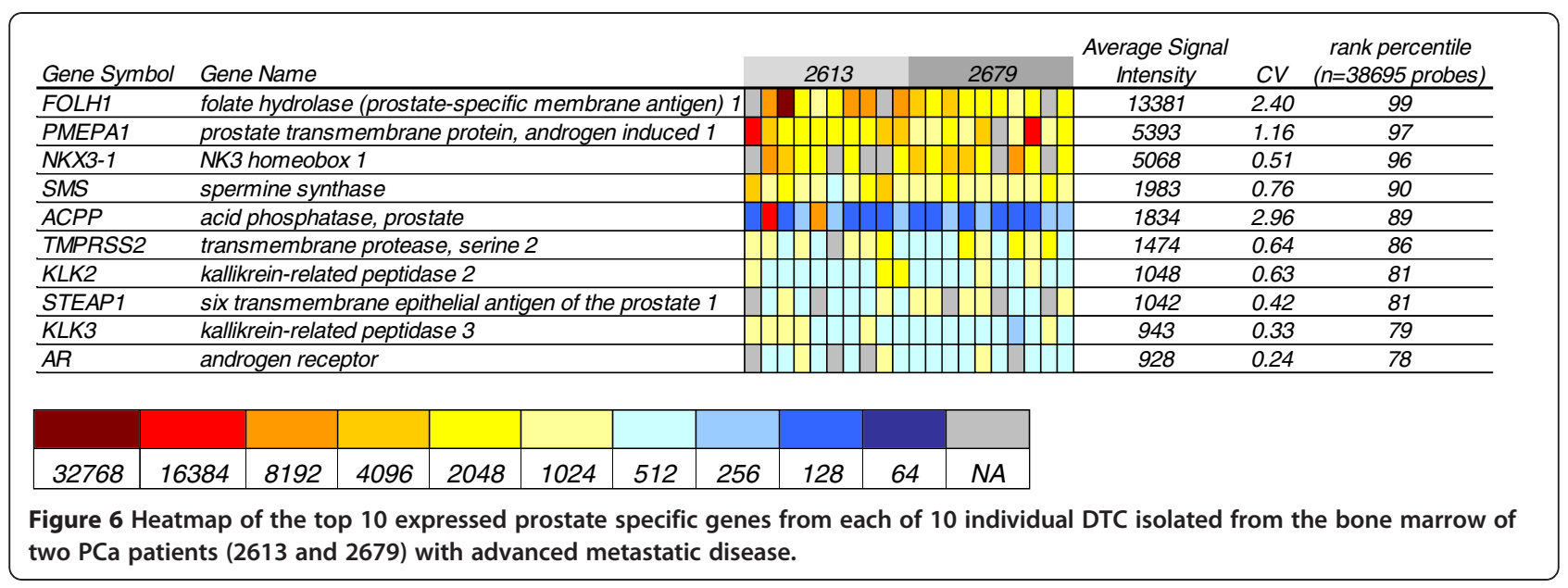

relevant gene expression profile from individual cells isolated from clinical biospecimens.

The caveats associated with the isolation of single cells from patient samples include: warm ischemia time, cell viability, and the RNase activity in cancer cells in vivo relative to somatic cells. While these parameters could all impact the quality of samples and therefore limit the transcriptomic profile observed, we have shown that a transcriptomic profile can be obtained from patient specimens. Additionally, examining tumor cell heterogeneity requires a greater number of single-cell samples to be analyzed from each patient; e.g., when profiling 10 cells from one patient, sampling 10 patients would now require 100 samples for analysis. This highlights the importance of a validated and efficient commercially available detection methodology that limits sample-to-sample variability and sample quality. A future challenge to increase the utility of this approach will be to automate the process of isolating cells of interest, possibly using microfluidic devices [21], and to provide a stage for direct RNA amplification or RNA-seq methodologies.

\section{Conclusions}

While the amount of material available for amplification is restrictive limiting the number of positive probes on the single-cell arrays, we found good correlation between relative signal intensities of probes detected on single-cell arrays when compared to both 5- and 10-cell arrays. Therefore, obtaining a transcriptomic profile from a single cell in culture and from patient biospecimens using commercially available technology is feasible and potentially useful. In its current form this methodology can be used for research purposes; however, due to the dramatic potential increase in sample number per patient, the successful combination of this technology with novel highthroughput procedures will be required for clinical utility.

\section{Additional files}

Additional file 1: Table S1. Primer sequences, amplicon length, possible splice variants, $3^{\prime}$ bias, and primer specificity of the 10 genes examined by RT-qPCR in this study.

Additional file 2: Table S2. Quantitative RT-PCR of 4 housekeeping genes (ACTB, GAPDH, YWHAZ, and GAPDH) for each of the 10 single-, 5-, and 10-cell samples. * Indicates removed from analysis based on quality control. NTC = No template control; na = no amplification detected after 46 cycles; $10 \mathrm{pg}$ and $100 \mathrm{pg}$ represents C4-2B total RNA from the same original culture.

Additional file 3: Table S3. M ratio pair-wise correlation analysis for one cell. Based on the Dixon test for the correlation coefficients, the $p$ value for testing outliers is 0.457 . Using $p$-value $=0.05$ as cut-off. Array data: The microarray data for these experiments have been deposited in the Gene Expression Omnibus database (available at http://www.ncbi. nlm.nih.gov/geo) under accession number GSE38416.

Additional file 4: Table S4. Gene Set Enrichment Analysis (GSEA) of single-, 5-, and 10-cell samples. GO = Gene Ontology; NES = Normalized Enrichment Score. The enrichment score reflects the degree to which the gene set is overrepresented at the extremes of the entire ranked list $(n=925)$.

\section{Authors' contributions}

$C M, R L V, C W, I C, R C$, and $B L$ conceived and designed the experiments. IC, RC, JX, RG, SC, CW, BL and CM analyzed the data. CW, IC, RC, BL, JX, SC, RLV, and $C M$ wrote the manuscript. CW, RC, BL, SRL and CG performed the experiments. PHL, BM, PN provided materials and input into the project's direction. All authors read and approved the final manuscript.

\section{Acknowledgements}

We would like to thank the patients who donated bone marrow aspirates that made this work possible. Additionally we would like to thank Marty Kinnunen, Lisha Brown, and Jennilee Kho for their contributions. These studies were supported by the NIH RC1 CA144825-01 ARRA Challenge and P01CA85859. CW was supported by the Ruth L. Kirschstein National Research Service Award (NRSA) Training Grant (T32). This material is the result of work supported by resources from the VA Puget Sound Health Care System, Seattle, Washington (RLV is a Research Career Scientist, PHL is a Staff Physician). CM is a recipient of a Career Development Award from the Pacific Northwest Prostate Cancer SPORE P50CA097186.

\section{Author details}

${ }^{1}$ Department of Urology, University of Washington, Seattle, WA, USA. ${ }^{2}$ Fred Hutchinson Cancer Research Center, Seattle, WA, USA. ${ }^{3}$ Department of 
Veterans Affairs Medical Center, Seattle, WA, USA. ${ }^{4}$ Department of Medicine, University of Washington, Seattle, WA, USA. ${ }^{5}$ Genitourinary Cancer Research Laboratory, Department of Urology, University of Washington, Box 356510, Seattle, WA 98195, USA.

Received: 7 September 2012 Accepted: 11 February 2013 Published: 16 February 2013

\section{References}

1. Holcomb IN, Grove DI, Kinnunen M, Friedman CL, Gallaher IS, Morgan TM, Sather CL, Delrow JJ, Nelson PS, Lange PH, Ellis WJ, True LD, Young JM, Hsu $L$, Trask BJ, Vessella RL: Genomic alterations indicate tumor origin and varied metastatic potential of disseminated cells from prostate cancer patients. Cancer Res 2008, 68:5599-5608.

2. Morgan TM, Lange PH, Porter MP, Lin DW, Ellis WJ, Gallaher IS, Vessella RL: Disseminated tumor cells in prostate cancer patients after radical prostatectomy and without evidence of disease predicts biochemical recurrence. Clin Cancer Res 2009, 15:677-683.

3. Oberneder R, Riesenberg R, Kriegmair M, Bitzer U, Klammert R, Schneede $P$, Hofstetter A, Riethmuller G, Pantel K: Immunocytochemical detection and phenotypic characterization of micrometastatic tumour cells in bone marrow of patients with prostate cancer. Urol Res 1994, 22:3-8.

4. Riethdorf S, Wikman H, Pantel K: Review: Biological relevance of disseminated tumor cells in cancer patients. Int J Cancer 2008, 123:1991-2006.

5. Pfitzenmaier J, Ellis WJ, Arfman EW, Hawley S, McLaughlin PO, Lange PH, Vessella RL: Telomerase activity in disseminated prostate cancer cells. BJU Int 2006, 97:1309-1313.

6. Shiozawa Y, Pedersen EA, Patel LR, Ziegler AM, Havens AM, Jung Y, Wang J, Zalucha S, Loberg RD, Pienta KJ, Taichman RS: GAS6/AXL axis regulates prostate cancer invasion, proliferation, and survival in the bone marrow niche. Neoplasia 2010, 12:116-127.

7. Wikman $H$, Vessella R, Pantel K: Cancer micrometastasis and tumour dormancy. APMIS 2008, 116:754-770.

8. Hartmann $\mathrm{CH}$, Klein CA: Gene expression profiling of single cells on largescale oligonucleotide arrays. Nucleic Acids Res 2006, 34:e143.

9. Klein CA, Seidl S, Petat-Dutter K, Offner S, Geigl JB, Schmidt-Kittler O, Wendler N, Passlick B, Huber RM, Schlimok G, Baeuerle PA, Riethmuller G: Combined transcriptome and genome analysis of single micrometastatic cells. Nat Biotechnol 2002, 20:387-392

10. Kurimoto $K$, Yabuta $Y$, Ohinata $Y$, Ono $Y$, Uno KD, Yamada RG, Ueda HR, Saitou M: An improved single-cell cDNA amplification method for efficient high-density oligonucleotide microarray analysis. Nucleic Acids Res 2006, 34:e42.

11. Brady G, Barbara M, Iscove NM: Representative in vitro CDNA amplification from individual hemopoietic cells and colonies. Meth Mol Cell Biol 1990, 2:17-25.

12. Ellis WJ, Pfitzenmaier J, Colli J, Arfman E, Lange PH, Vessella RL: Detection and isolation of prostate cancer cells from peripheral blood and bone marrow. Urology 2003, 61:277-281.

13. Dixon WJ: Analysis of extreme values. Ann Math Stat 1950, 21:488-506.

14. Smyth GK: Limma: linear models for microarray data. In Bioinformatics and Computational Biology Solutions using $R$ and Bioconductor. Edited by Gentleman R, Carey V, Dudoit S, Irizarry R, Huber W. New York: Springer; 2005:397-420.

15. Subramanian A, Tamayo P, Mootha VK, Mukherjee S, Ebert BL, Gillette MA, Paulovich A, Pomeroy SL, Golub TR, Lander ES, Mesirov JP: Gene set enrichment analysis: a knowledge-based approach for interpreting genome-wide expression profiles. Proc Natl Acad Sci U S A 2005, 102:15545-15550

16. Mootha VK, Lindgren CM, Eriksson KF, Subramanian A, Sihag S, Lehar J, Puigserver P, Carlsson E, Ridderstrale M, Laurila E, Houstis N, Daly MJ, Patterson N, Mesirov JP, Golub TR, Tamayo P, Spiegelman B, Lander ES, Hirschhorn JN, Altshuler D, Groop LC: PGC-1alpha-responsive genes involved in oxidative phosphorylation are coordinately downregulated in human diabetes. Nat Genet 2003, 34:267-273.

17. Islam S, Kjallquist U, Moliner A, Zajac P, Fan JB, Lonnerberg P, Linnarsson S: Characterization of the single-cell transcriptional landscape by highly multiplex RNA-seq. Genome Res 2011, 21:1160-1167.

18. Cann GM, Gulzar ZG, Cooper S, Li R, Luo S, Tat M, Stuart S, Schroth G, Srinivas S, Ronaghi M, Brooks JD, Talasaz AH: mRNA-Seq of single prostate cancer circulating tumor cells reveals recapitulation of gene expression and pathways found in prostate cancer. PLoS One 2012, 7:e49144.

19. van de Stolpe A, Pantel K, Sleijfer S, Terstappen LW, den Toonder JM: Circulating tumor cell isolation and diagnostics: toward routine clinical use. Cancer Res 2011, 71:5955-5960.

20. Economos C, Morrissey C, Vessella RL: Circulating tumor cells as a marker of response: implications for determining treatment efficacy and evaluating new agents. Curr Opin Urol 2012, 22:190-196.

21. Melin J, Quake SR: Microfluidic large-scale integration: the evolution of design rules for biological automation. Annu Rev Biophys Biomol Struct 2007, 36:213-231.

doi:10.1186/1471-2199-14-6

Cite this article as: Welty et al:: Single cell transcriptomic analysis of prostate cancer cells. BMC Molecular Biology 2013 14:6.

\section{Submit your next manuscript to BioMed Central and take full advantage of:}

- Convenient online submission

- Thorough peer review

- No space constraints or color figure charges

- Immediate publication on acceptance

- Inclusion in PubMed, CAS, Scopus and Google Scholar

- Research which is freely available for redistribution 